\title{
EVALUATION OF DETERMINANT OF'11TH ORDER IN THE THEORY OF PARTITIONS
}

S. MANZUR HUSSAIN

(received 16 May 1960)

Determinants [1] of 5th and 7th orders have already been discussed in connection with the Theorems 4 and 5 of Atkin and Swinnerton-Dyer [2]. The determinant under consideration occurs in the investigation of Dyson's rank function for $q=11$ given by Atkin and Hussain [3]. As regards the notation it may be mentioned that the author has adopted the same as that of Atkin and Hussain [3].

Let

$$
\begin{array}{llrl}
a & =-x^{-4} P(2) / P(1), & b & x^{-5} P(4) / P(2) \\
c & =x^{2} P(3) / P(4), & d=-x^{-3} P(5) / P(3) . \\
e & =-x^{10} P(1) / P(5), & &
\end{array}
$$

Let $\Delta_{11} / y^{5}$ be equivalent to

$$
\left|\begin{array}{lllllllllll}
b & 0 & 0 & 0 & c x^{4} & 0 & x^{6} & e x^{7} & 0 & d x^{9} & a x^{10} \\
a x^{-1} & b & 0 & 0 & 0 & c x^{4} & 0 & x^{6} & e x^{7} & 0 & d x^{9} \\
d x^{-2} & a x^{-1} & b & 0 & 0 & 0 & c x^{4} & 0 & x^{6} & e x^{7} & 0 \\
0 & d x^{-2} & a x^{-1} & b & 0 & 0 & 0 & c x^{4} & 0 & x^{6} & e x^{7} \\
e x^{-4} & 0 & d x^{-2} & a x^{-1} & b & 0 & 0 & 0 & c x^{4} & 0 & x^{6} \\
x^{-5} & e x^{-4} & 0 & d x^{-2} & a x^{-1} & b & 0 & 0 & 0 & c x^{4} & 0 \\
0 & x^{-5} & e x^{-4} & 0 & d x^{-2} & a x^{-1} & b & 0 & 0 & 0 & c x^{4} \\
c x^{-7} & 0 & x^{-5} & e x^{-4} & 0 & d x^{-2} & a x^{-1} & b & 0 & 0 & 0 \\
0 & c x^{-7} & 0 & x^{-5} & e x^{-4} & 0 & d x^{-2} & a x^{-1} & b & 0 & 0 \\
0 & 0 & c x^{-7} & 0 & x^{-5} & e x^{-4} & 0 & d x^{-2} & a x^{-1} & b & 0 \\
0 & 0 & 0 & c x^{-7} & 0 & x^{-5} & e x^{-4} & 0 & d x^{-2} & a x^{-1} & b
\end{array}\right|
$$

Then

$$
\Delta_{11} / y^{5}=\lambda \mu-17 \lambda^{2}-108 \mu+346 \lambda-131 .
$$

Multiplying the $(i+1)$ th row by $x^{i} w_{r}^{i}$ where $i=0$ to 10 and adding it is found that $\left(a w_{r}+b+c w_{r}^{2}+d w_{r}^{2}+e w_{r}^{4}+w w_{r}^{5}\right)$ is a common factor where $w_{r}$ is an 11th root of unity. 
Thus

$$
\begin{aligned}
\Delta_{11} / y^{5} & =\prod_{r=1}^{11}\left(a w w_{r}+b+c w w_{r}^{7}+d w w_{r}^{2}+e v w_{r}^{15}+w w_{r}^{5}\right), \\
& =\prod_{r=1}^{11}\left(a w w_{r}^{-4}+b w_{r}^{-5}+c w w_{r}^{2}+d w w_{r}^{-3}+e w w_{r}^{10}+1\right) ; \\
& =y^{-5} f^{12}(y) / f^{12}\left(y^{11}\right) \\
& =\lambda \mu-17 \lambda^{2}-108 \mu+364 \lambda-131
\end{aligned}
$$

Vide the results (11.4) to (11.7) of Atkin and Hussain (3).

\section{References}

[1] Proceedings of the Pakistan Statistical Association 6 (1957) 27-28

[2] A.O.L. Atkin and P. Swinnerton-Dyer, Some properties of Partition, Proc. London Math. Society (3) 4 (1953) pp. 84-106.

[3] A.O.L. Atkin and S. M. Hussain, some properties of Partition (2) Transactions of the American Math, Society, 89, (1958) 184-200.

University of the Panajb,

Lahore, (West Pakistan). 